\title{
Baryon Decuplet to Octet Electromagnetic Transitions in Quenched and Partially Quenched Chiral Perturbation Theory
}

\author{
Daniel Arndt* and Brian C. Tiburzit \\ Department of Physics, Box 351560, \\ University of Washington, Seattle, WA 98195-1560, USA
}

(Dated: October 30, 2018)

\begin{abstract}
We calculate baryon decuplet to octet electromagnetic transition form factors in quenched and partially quenched chiral perturbation theory. We work in the isospin limit of $S U(3)$ flavor, up to next-to-leading order in the chiral expansion, and to leading order in the heavy baryon expansion. Our results are necessary for proper extrapolation of lattice calculations of these transitions. We also derive expressions for the case of $S U(2)$ flavor away from the isospin limit.
\end{abstract}

*arndt@phys.washington.edu

bctiburz@phys.washington.edu

Typeset by REVTEX 


\section{INTRODUCTION}

The study of the baryon decuplet to octet electromagnetic transitions provides important insight into the strongly interacting regime of QCD. Spin-parity selection rules for these transitions allow for magnetic dipole (M1), electric quadrupole (E2), and Coulumb quadrupole (C2) amplitudes. Understanding these amplitudes, both in theory and experiment, gives insight into the ground state wavefunctions of the lowest lying baryons. For example, in the transition of the $\Delta(1232)$ to the nucleon, if both baryon wavefunctions are spherically symmetric then the E2 and C2 amplitudes vanish. Experimentally, M1 is seen to be the dominant amplitude. However, recent experimental measurements of the quadrupole amplitudes in the $\Delta \rightarrow N \gamma$ transition [1, 2] show that the quadrupole amplitudes E2 and C2 are likely non-zero. This has revitalized the discussion as to the mechanism for deformation of the baryons. Although we expect more experimental data in the future, progress will be slower for the remaining transitions as the experimental difficulties are significant.

First-principle lattice QCD calculations of these matrix elements can provide a theoretical explanation of these experimental results. In fact, the experimental difficulties may force us to rely on lattice data for the non-nucleonic transitions. Recently several such lattice calculations [3, 4], which improve upon an earlier one [5], have appeared. Although these calculations still largely employ the quenched approximation of QCD, we expect partially quenched calculations to be performed in the near future. Unfortunately now and foreseeably, these lattice calculations cannot be performed with the physical masses of the light quarks as the calculation time would be prohibitively long. Therefore, to make physical predictions, it is necessary to extrapolate from the heavier quark masses used on the lattice (currently on the order of the strange quark mass) down to the physical light quark masses. Chiral perturbation theory $(\chi \mathrm{PT})$ provides model-independent input for the behavior of observables as a function of quark masses.

For lattice calculations that use the quenched approximation of QCD (QQCD), where the fermion determinant that arises from the path integral is set equal to one, quenched chiral perturbation theory $(\mathrm{Q} \chi \mathrm{PT})[6,6$, 8, 9, 10, 11, 12] has been developed to aid in the extrapolation. The problem with the quenched approximation is that the Goldstone boson singlet, the $\eta^{\prime}$, which is heavy in QCD, remains light in QQCD and must be retained in $\mathrm{Q} \chi \mathrm{PT}$, requiring the addition of new operators and hence new low-energy constants in the Lagrangian. In general, the low-energy constants appearing in the $\mathrm{Q} \chi \mathrm{PT}$ Lagrangian are unrelated to those in $\chi \mathrm{PT}$ and extrapolated quenched lattice data is unrelated to QCD. In fact, several examples show that the behavior of meson loops near the chiral limit is frequently misrepresented in Q $\chi \mathrm{PT}[13,14,15,16,17,18]$.

These problems of QQCD can be remedied by using partially quenched lattice QCD (PQQCD). Unlike QQCD, where the masses of quarks not connected to external sources are set to infinity, these "sea quark" masses are kept finite in PQQCD. The masses of the sea quarks can be varied independently of the valence quark masses; usually they are chosen to be heavier. By keeping the sea quarks as dynamical degrees of freedom, the fermion determinant is no longer equal to one and needs to be computed. However, by efficaciously giving the sea quarks larger masses it is much less costly to calculate. Moreover, since PQQCD retains a $U(1)_{A}$ anomaly, the equivalent to the singlet field in QCD is heavy (on the order of the chiral symmetry breaking scale $\Lambda_{\chi}$ ) and can be integrated out [19, 20]. As a consequence, the lowenergy constants appearing in partially quenched chiral perturbation theory (PQ $\chi \mathrm{PT})$ 19, 20, 21, 22, 23, 24, 25, 26], which is the low-energy effective theory of PQQCD, are the 
same as those appearing in $\chi \mathrm{PT}$. By fitting PQ $\chi \mathrm{PT}$ to partially quenched lattice data, one can determine these constants and actually make physical predictions for QCD. PQ $\chi \mathrm{PT}$ has been used recently to study heavy meson [27] and octet baryon observables [17, 28, 29, 30, 31]. The available lattice calculations for the $\Delta \rightarrow N \gamma$ transition [3, 4] use the quenched approximation; there are currently no partially quenched simulations. However, given the recent progress that lattice gauge theory has made in the one-hadron sector and the prospect of simulations in the two-hadron sector [32, 33, 34, 35, 36], we expect to see partially quenched calculations of these form factors in the near future.

This paper is organized as follows. First, in Section $\llbracket$ we briefly review PQ $\chi$ PT including the treatment of the baryon octet and decuplet in the heavy baryon approximation [37, 38]. Since we will use the conventions used in our recent related work on the octet and decuplet baryons [17, 18] we will keep this section brief. In Section [II we calculate baryon decuplet to octet transition form factors in both $\mathrm{Q} \chi \mathrm{PT}$ and PQ $\chi \mathrm{PT}$ up to next-to-leading (NLO) order in the chiral expansion and keep contributions to lowest order in the heavy baryon mass, $M_{B}$. These calculations are done in the isospin limit of $S U(3)$ flavor. For completeness we also provide the PQ $\chi \mathrm{PT}$ results for the transitions using the $S U(2)$ chiral Lagrangian with non-degenerate quarks in the Appendix. In Section [V we conclude.

\section{II. $\mathbf{P Q} \chi \mathbf{P T}$}

In PQQCD the quark part of the Lagrangian is written as [19, 20, 21, 22, 23, 24, 25, 26]

$$
\mathcal{L}=\sum_{j, k=1}^{9} \bar{Q}_{j}\left(i \not D-m_{Q}\right)_{j k} Q_{k}
$$

that differs from the QCD $S U(3)$ flavor Lagrangian by the inclusion of three bosonic ghost quarks, $\tilde{u}, \tilde{d}$, and $\tilde{s}$, and three fermionic sea quarks, $j, l$, and $r$, in addition to the fermionic light valence quarks $u, d$, and $s$. These nine quarks are in the fundamental representation of the graded group $S U(6 \mid 3)$ [39, 40, 41] and have been accommodated in the nine-component vector

$$
Q=(u, d, s, j, l, r, \tilde{u}, \tilde{d}, \tilde{s})
$$

that obeys the graded equal-time commutation relation

$$
Q_{i}^{\alpha}(\mathbf{x}) Q_{j}^{\beta^{\dagger}}(\mathbf{y})-(-1)^{\eta_{i} \eta_{j}} Q_{j}^{\beta^{\dagger}}(\mathbf{y}) Q_{i}^{\alpha}(\mathbf{x})=\delta^{\alpha \beta} \delta_{i j} \delta^{3}(\mathbf{x}-\mathbf{y})
$$

where $\alpha$ and $\beta$ are spin and $i$ and $j$ are flavor indices. The graded equal-time commutation relations for two $Q$ 's and two $Q^{\dagger}$ 's can be written analogously. The grading factor

$$
\eta_{k}=\left\{\begin{array}{l}
1 \text { for } k=1,2,3,4,5,6 \\
0 \text { for } k=7,8,9
\end{array}\right.
$$

takes into account the different statistics for fermionic and bosonic quarks. The quark mass matrix is given by

$$
m_{Q}=\operatorname{diag}\left(m_{u}, m_{d}, m_{s}, m_{j}, m_{l}, m_{r}, m_{u}, m_{d}, m_{s}\right)
$$

so that diagrams with closed ghost quark loops cancel those with valence quarks. Effects of virtual quark loops are, however, present due to the contribution of the finite-mass sea quarks. 
As has been recently realized [42], the light quark electric charge matrix $\mathcal{Q}$ is not uniquely defined in PQQCD and the only constraint one imposes is for $\mathcal{Q}$ to have vanishing supertrace so that, as in QCD, no new operators involving the singlet component are introduced. Following [28] we use

$$
\mathcal{Q}=\operatorname{diag}\left(\frac{2}{3},-\frac{1}{3},-\frac{1}{3}, q_{j}, q_{l}, q_{r}, q_{j}, q_{l}, q_{r}\right) .
$$

QCD is recovered in the limit $m_{j} \rightarrow m_{u}, m_{l} \rightarrow m_{d}$, and $m_{r} \rightarrow m_{s}$ independently of the $q$ 's.

For massless quarks, the Lagrangian in Eq. (1) exhibits a graded symmetry $S U(6 \mid 3)_{L} \otimes$ $S U(6 \mid 3)_{R} \otimes U(1)_{V}$ that is assumed to be spontaneously broken down to $S U(6 \mid 3)_{V} \otimes U(1)_{V}$. The low-energy effective theory of PQQCD that emerges by expanding about the physical vacuum state is PQ $\chi \mathrm{PT}$. The dynamics of the emerging 80 pseudo-Goldstone mesons can be described at lowest order in the chiral expansion by the $\mathcal{O}\left(E^{2}\right)$ Lagrangian $^{1}$

$$
\mathcal{L}=\frac{f^{2}}{8} \operatorname{str}\left(D^{\mu} \Sigma^{\dagger} D_{\mu} \Sigma\right)+\lambda \operatorname{str}\left(m_{Q} \Sigma+m_{Q}^{\dagger} \Sigma^{\dagger}\right)+\alpha \partial^{\mu} \Phi_{0} \partial_{\mu} \Phi_{0}-\mu_{0}^{2} \Phi_{0}^{2}
$$

where

$$
\Sigma=\exp \left(\frac{2 i \Phi}{f}\right)=\xi^{2}, \quad \Phi=\left(\begin{array}{cc}
M & \chi^{\dagger} \\
\chi & \tilde{M}
\end{array}\right),
$$

$f=132 \mathrm{MeV}$, and the gauge-covariant derivative is $D_{\mu} \Sigma=\partial_{\mu} \Sigma+i e \mathcal{A}_{\mu}[\mathcal{Q}, \Sigma] . \operatorname{The} \operatorname{str}()$ denotes a supertrace over flavor indices. The $M, \tilde{M}$, and $\chi$ are matrices of pseudo-Goldstone bosons with quantum numbers of $q \bar{q}$ pairs, pseudo-Goldstone bosons with quantum numbers of $\tilde{q} \bar{q}$ pairs, and pseudo-Goldstone fermions with quantum numbers of $\tilde{q} \bar{q}$ pairs, respectively. $\Phi$ is defined in the quark basis and normalized such that $\Phi_{12}=\pi^{+}$(see, for example, [28]). Upon expanding the Lagrangian in (7) one finds that to lowest order the mesons with quark content $Q \bar{Q}^{\prime}$ are canonically normalized when their masses are given by $m_{Q Q^{\prime}}^{2}=$ $\frac{4 \lambda}{f^{2}}\left(m_{Q}+m_{Q^{\prime}}\right)$.

The flavor singlet field given by $\Phi_{0}=\operatorname{str}(\Phi) / \sqrt{6}$ is, in contrast to the $\mathrm{Q} \chi \mathrm{PT}$ case, rendered heavy by the $U(1)_{A}$ anomaly and can therefore be integrated out in $\chi \mathrm{PT}$. Analogously its mass $\mu_{0}$ can be taken to be on the order of the chiral symmetry breaking scale, $\mu_{0} \rightarrow \Lambda_{\chi}$. In this limit the flavor singlet propagator becomes independent of the coupling $\alpha$ and deviates from a simple pole form [19, 20].

Just as there are mesons in PQQCD with quark content $\bar{Q}_{i} Q_{j}$ that contain valence, sea, and ghost quarks, there are baryons with quark compositions $Q_{i} Q_{j} Q_{k}$ that contain all three types of quarks. To this end, one decomposes the irreducible representations of $S U(6 \mid 3)_{V}$ into irreducible representations of $S U(3)_{\mathrm{val}} \otimes S U(3)_{\mathrm{sea}} \otimes S U(3)_{\text {ghost }} \otimes U(1)$. The method to construct the octet baryons is to use the interpolating field

$$
\mathcal{B}_{i j k}^{\gamma} \sim\left(Q_{i}^{\alpha, a} Q_{j}^{\beta, b} Q_{k}^{\gamma, c}-Q_{i}^{\alpha, a} Q_{j}^{\gamma, c} Q_{k}^{\beta, b}\right) \epsilon_{a b c}\left(C \gamma_{5}\right)_{\alpha \beta}
$$

The spin-1/2 baryon octet $B_{i j k}=\mathcal{B}_{i j k}$, where the indices $i, j$, and $k$ are restricted to $1-3$, is contained as a $(\mathbf{8}, \mathbf{1}, \mathbf{1})$ of $S U(3)_{\text {val }} \otimes S U(3)_{\text {sea }} \otimes S U(3)_{\text {ghost }}$ in the $\mathbf{2 4 0}$ representation. The

\footnotetext{
${ }^{1}$ Here, $E \sim p, m_{\pi}$ where $p$ is an external momentum.
} 
octet baryons, written in the familiar two-index notation

$$
B=\left(\begin{array}{ccc}
\frac{1}{\sqrt{6}} \Lambda+\frac{1}{\sqrt{2}} \Sigma^{0} & \Sigma^{+} & p \\
\Sigma^{-} & \frac{1}{\sqrt{6}} \Lambda-\frac{1}{\sqrt{2}} \Sigma^{0} & n \\
\Xi^{-} & \Xi^{0} & -\frac{2}{\sqrt{6}} \Lambda
\end{array}\right),
$$

are embedded in $B_{i j k}$ as [12]

$$
B_{i j k}=\frac{1}{\sqrt{6}}\left(\epsilon_{i j l} B_{k l}+\epsilon_{i k l} B_{j l}\right) .
$$

The remaining baryon states needed for our calculation have at most one ghost or one sea quark and have been constructed explicitly in [28].

Similarly, the familiar spin-3/2 decuplet baryons are embedded in the $\mathbf{1 6 5}$. Here, one uses the interpolating field

$$
\mathcal{T}_{i j k}^{\alpha, \mu} \sim\left(Q_{i}^{\alpha, a} Q_{j}^{\beta, b} Q_{k}^{\gamma, c}+Q_{i}^{\beta, b} Q_{j}^{\gamma, c} Q_{k}^{\alpha, a}+Q_{i}^{\gamma, c} Q_{j}^{\alpha, a} Q_{k}^{\beta, b}\right) \epsilon_{a b c}\left(C \gamma^{\mu}\right)_{\beta \gamma}
$$

that describes the 165 dimensional representation of $S U(6 \mid 3)_{V}$. The decuplet baryons $T_{i j k}$ are then readily embedded in $\mathcal{T}$ by construction: $T_{i j k}=\mathcal{T}_{i j k}$, where the indices $i$, $j$, and $k$ are restricted to $1-3$. They transform as a $(\mathbf{1 0}, \mathbf{1}, \mathbf{1})$ under $S U(3)_{\text {val }} \otimes S U(3)_{\text {sea }} \otimes S U(3)_{\text {ghost }}$. Because of Eqs. (3) and (12), $T_{i j k}$ is a totally symmetric tensor. Our normalization convention is such that $T_{111}=\Delta^{++}$. For the spin-3/2 baryons consisting of two valence and one ghost quark or two valence and one sea quark, we use the states constructed in [28].

At leading order in the heavy baryon expansion, the free Lagrangian for the $\mathcal{B}_{i j k}$ and $\mathcal{T}_{i j k}$ is given by [12]

$$
\begin{aligned}
\mathcal{L}= & i(\overline{\mathcal{B}} v \cdot \mathcal{D B})+2 \alpha_{M}\left(\overline{\mathcal{B}} \mathcal{B} \mathcal{M}_{+}\right)+2 \beta_{M}\left(\overline{\mathcal{B}} \mathcal{M}_{+} \mathcal{B}\right)+2 \sigma_{M}(\overline{\mathcal{B}} \mathcal{B}) \operatorname{str}\left(\mathcal{M}_{+}\right) \\
& -i\left(\overline{\mathcal{T}}^{\mu} v \cdot \mathcal{D} \mathcal{T}_{\mu}\right)+\Delta\left(\overline{\mathcal{T}}^{\mu} \mathcal{T}_{\mu}\right)+2 \gamma_{M}\left(\overline{\mathcal{T}}^{\mu} \mathcal{M}_{+} \mathcal{T}_{\mu}\right)-2 \bar{\sigma}_{M}\left(\overline{\mathcal{T}}^{\mu} \mathcal{T}_{\mu}\right) \operatorname{str}\left(\mathcal{M}_{+}\right)
\end{aligned}
$$

where $\mathcal{M}_{+}=\frac{1}{2}\left(\xi^{\dagger} m_{Q} \xi^{\dagger}+\xi m_{Q} \xi\right)$. The brackets in (13) are shorthands for field bilinear invariants originally employed in [12]. The Lagrangian describing the relevant interactions of the $\mathcal{B}_{i j k}$ and $\mathcal{T}_{i j k}$ with the pseudo-Goldstone mesons is

$$
\mathcal{L}=2 \alpha\left(\overline{\mathcal{B}} S^{\mu} \mathcal{B} A_{\mu}\right)+2 \beta\left(\overline{\mathcal{B}} S^{\mu} A_{\mu} \mathcal{B}\right)+\sqrt{\frac{3}{2}} \mathcal{C}\left[\left(\overline{\mathcal{T}}^{\nu} A_{\nu} \mathcal{B}\right)+\text { h.c. }\right]+2 \mathcal{H}\left(\overline{\mathcal{T}}^{\nu} S^{\mu} A_{\mu} \mathcal{T}_{\nu}\right)
$$

where the axial-vector and vector meson fields $A^{\mu}$ and $V^{\mu}$ are defined in analogy to those in QCD, $A^{\mu}=\frac{i}{2}\left(\xi \partial^{\mu} \xi^{\dagger}-\xi^{\dagger} \partial^{\mu} \xi\right)$ and $V^{\mu}=\frac{1}{2}\left(\xi \partial^{\mu} \xi^{\dagger}+\xi^{\dagger} \partial^{\mu} \xi\right)$. The latter appears in Eq. (13) in the covariant derivatives of $\mathcal{B}_{i j k}$ and $\mathcal{T}_{i j k}$ that both have the form

$$
\left(\mathcal{D}^{\mu} \mathcal{B}\right)_{i j k}=\partial^{\mu} \mathcal{B}_{i j k}+\left(V^{\mu}\right)_{i l} \mathcal{B}_{l j k}+(-)^{\eta_{i}\left(\eta_{j}+\eta_{m}\right)}\left(V^{\mu}\right)_{j m} \mathcal{B}_{i m k}+(-)^{\left(\eta_{i}+\eta_{j}\right)\left(\eta_{k}+\eta_{n}\right)}\left(V^{\mu}\right)_{k n} \mathcal{B}_{i j n}
$$

By restricting the indices of $\mathcal{B}_{i j k}$ to $i, j, k=1,2,3$ one can relate the constants $\alpha$ and $\beta$ to $D$ and $F$ that are used for the $S U(3)_{\text {val }}$ analogs of these terms in QCD and finds

$$
\alpha=\frac{2}{3} D+2 F, \quad \beta=-\frac{5}{3} D+F,
$$

while $\mathcal{C}$ and $\mathcal{H}$ are the constants of QCD. 


\section{BARYON DECUPLET TO OCTET TRANSITION}

The electromagnetic baryon decuplet to octet transitions have been investigated previously in $\chi \mathrm{PT}[43,44,45$, 46]. Very recently there also has been renewed interest in these transitions in the large $N_{c}$ limit of QCD [47]. Here we calculate these transitions in PQ $\chi \mathrm{PT}$ and $\mathrm{Q} \chi \mathrm{PT}$. While we have reviewed PQ $\chi \mathrm{PT}$ briefly in the last section and our recent papers [17, 18], for $\mathrm{Q} \chi \mathrm{PT}$ we refer the reader to the literature [6, 7, 8, 9, 10, 11, 12].

Using the heavy baryon formalism [37, 38], transition matrix elements of the electromagnetic current $J^{\rho}$ between a decuplet baryon with momentum $p^{\prime}$ and an octet baryon with momentum $p$ can be parametrized as

$$
\left\langle\bar{B}(p)\left|J^{\rho}\right| T\left(p^{\prime}\right)\right\rangle=\bar{u}(p) \mathcal{O}^{\rho \mu} u_{\mu}\left(p^{\prime}\right)
$$

where $u_{\mu}(p)$ is a Rarita-Schwinger spinor for an on-shell decuplet baryon satisfying $v^{\mu} u_{\mu}(p)=$ 0 and $S^{\mu} u_{\mu}(p)=0$. The tensor $\mathcal{O}^{\rho \mu}$ can be parametrized in terms of three independent, Lorentz invariant, dimensionless form factors [48]

$$
\begin{aligned}
\mathcal{O}^{\rho \mu}= & \frac{G_{1}\left(q^{2}\right)}{M_{B}}\left(q \cdot S g^{\mu \rho}-q^{\mu} S^{\rho}\right)+\frac{G_{2}\left(q^{2}\right)}{\left(2 M_{B}\right)^{2}}\left(q \cdot v g^{\mu \rho}-q^{\mu} v^{\rho}\right) S \cdot q \\
& +\frac{G_{3}\left(q^{2}\right)}{4 M_{B}^{2} \Delta}\left(q^{2} g^{\mu \rho}-q^{\mu} q^{\rho}\right) S \cdot q,
\end{aligned}
$$

where the momentum of the outgoing photon is $q=p^{\prime}-p$. Here we have adopted the normalization of the $G_{3}\left(q^{2}\right)$ form factor used in [46] so that the leading contributions to all three form factors are of order unity in the power counting.

Linear combinations of the above form factors at $q^{2}=0$ make the magnetic dipole, electric quadrupole, and Coulombic quadrupole moments,

$$
\begin{aligned}
G_{M 1}(0) & =\left(\frac{2}{3}-\frac{\Delta}{6 M_{B}}\right) G_{1}(0)+\frac{\Delta}{12 M_{B}} G_{2}(0) \\
G_{E 2}(0) & =\frac{\Delta}{6 M_{B}} G_{1}(0)+\frac{\Delta}{12 M_{B}} G_{2}(0) \\
G_{C 2}(0) & =\left(\frac{1}{3}+\frac{\Delta}{6 M_{B}}\right) G_{1}(0)+\left(\frac{1}{6}+\frac{\Delta}{6 M_{B}}\right) G_{2}(0)+\frac{1}{6} G_{3}(0) .
\end{aligned}
$$

\section{1. $P Q \chi P T$}

Let us first consider the transition form factors in PQ $\chi \mathrm{PT}$. Here, the leading tree-level contributions to the transition moments come from the dimension- 5 and dimension- 6 operators $^{2}$

$$
\mathcal{L}=\sqrt{\frac{3}{2}} \mu_{T} \frac{i e}{2 M_{B}}\left(\overline{\mathcal{B}} S^{\mu} \mathcal{Q} \mathcal{T}^{\nu}\right) F_{\mu \nu}+\sqrt{\frac{3}{2}} \mathbb{Q}_{T} \frac{e}{\Lambda_{\chi}^{2}}\left(\overline{\mathcal{B}} S^{\{\mu} \mathcal{Q} \mathcal{T}^{\nu\}}\right) v^{\alpha} \partial_{\mu} F_{\nu \alpha}
$$

where the action of $\{\ldots\}$ on Lorentz indices produces the symmetric traceless part of the tensor, viz., $\mathcal{O}^{\{\mu \nu\}}=\mathcal{O}^{\mu \nu}+\mathcal{O}^{\nu \mu}-\frac{1}{2} g^{\mu \nu} \mathcal{O}^{\alpha}{ }_{\alpha}$. Here the PQQCD low-energy constants $\mu_{T}$ and $\mathbb{Q}_{T}$ have the same numerical values as in QCD.

\footnotetext{
${ }^{2}$ We use $F_{\mu \nu}=\partial_{\mu} A_{\nu}-\partial_{\nu} A_{\mu}$.
} 

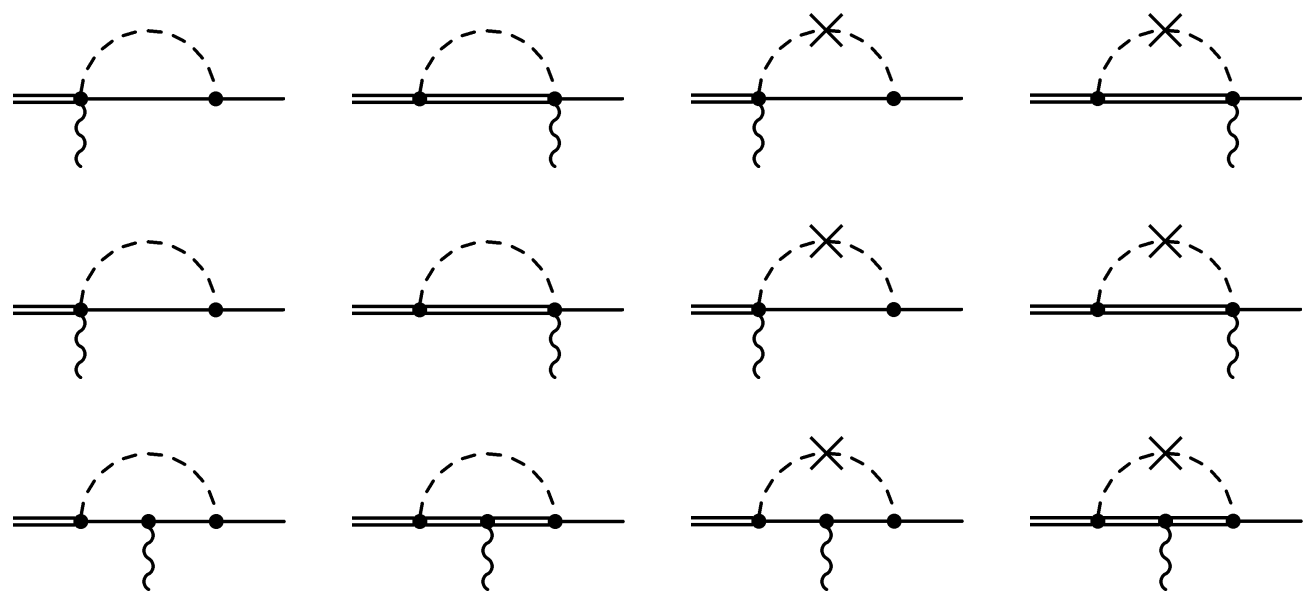

FIG. 1: Loop diagrams that contribute to the transition moments but are zero to the order we are working. A thin (thick) solid line denotes an octet (decuplet) baryon whereas a dashed line denotes a meson.
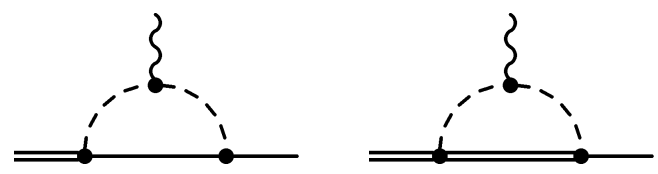

FIG. 2: Loop diagrams contributing to the transition moments.

The NLO contributions in the chiral expansion arise from the one-loop diagrams shown in Figs. (11) and (2). However, because of the constraints satisfied by the on-shell RaritaSchwinger spinors, the diagrams in Fig. (10) are all identically zero. Calculation of the diagrams in Fig. (2) gives

$$
\begin{aligned}
& G_{1}(0)= \frac{\mu_{T}}{2} \alpha+\frac{M_{B}}{\Lambda_{\chi}^{2}} 4 \mathcal{H C} \sum_{X} \beta_{X}^{T} \int_{0}^{1} d x\left(1-\frac{x}{3}\right)\left[x \Delta \log \frac{m_{X}^{2}}{\mu^{2}}-m_{X} \mathcal{R}\left(\frac{x \Delta}{m_{X}}\right)\right] \\
&-\frac{M_{B}}{\Lambda_{\chi}^{2}} 4 \mathcal{C}(D-F) \sum_{X} \beta_{X}^{B} \int_{0}^{1} d x(1-x)\left[x \Delta \log \frac{m_{X}^{2}}{\mu^{2}}+m_{X} \mathcal{R}\left(-\frac{x \Delta}{m_{X}}\right)\right], \\
& G_{2}(0)=\frac{M_{B}^{2}}{\Lambda_{\chi}^{2}}\left\{\begin{array}{l}
-4 \mathbb{Q}_{T} \alpha \\
\quad
\end{array}\right. \\
& \quad+16 \mathcal{H C} \sum_{X} \beta_{X}^{T} \int_{0}^{1} d x \frac{x(1-x)}{3}\left[\log \frac{m_{X}^{2}}{\mu^{2}}+\frac{x \Delta m_{X}}{m_{X}^{2}-x^{2} \Delta^{2}} \mathcal{R}\left(\frac{x \Delta}{m_{X}}\right)\right] \\
&\left.-16 \mathcal{C}(D-F) \sum_{X} \beta_{X}^{B} \int_{0}^{1} d x x(1-x)\left[\log \frac{m_{X}^{2}}{\mu^{2}}-\frac{x \Delta m_{X}}{m_{X}^{2}-x^{2} \Delta^{2}} \mathcal{R}\left(-\frac{x \Delta}{m_{X}}\right)\right]\right\}
\end{aligned}
$$


TABLE I: Tree-level coefficients $\alpha$ in $\chi \mathrm{PT}, \mathrm{Q} \chi \mathrm{PT}$, and PQ $\chi \mathrm{PT}$.

\begin{tabular}{c|c}
\hline \hline & $\alpha$ \\
\hline$\Delta \rightarrow N \gamma$ & $\frac{1}{\sqrt{3}}$ \\
$\Sigma^{*,+} \rightarrow \Sigma^{+} \gamma$ & $-\frac{1}{\sqrt{3}}$ \\
$\Sigma^{*, 0} \rightarrow \Sigma^{0} \gamma$ & $\frac{1}{2 \sqrt{3}}$ \\
$\Sigma^{*, 0} \rightarrow \Lambda \gamma$ & $-\frac{1}{2}$ \\
$\Sigma^{*,-} \rightarrow \Sigma^{-} \gamma$ & 0 \\
$\Xi^{*, 0} \rightarrow \Xi^{0} \gamma$ & $-\frac{1}{\sqrt{3}}$ \\
$\Xi^{*,-} \rightarrow \Xi^{-} \gamma$ & 0 \\
\hline \hline
\end{tabular}

and

$$
\begin{aligned}
G_{3}(0)= & -\frac{M_{B}^{2}}{\Lambda_{\chi}^{2}} 16\left[\mathcal{H C} \sum_{X} \beta_{X}^{T} \int_{0}^{1} d x \frac{x(1-x)}{3}\left(x-\frac{1}{2}\right) \frac{\Delta m_{X}}{m_{X}^{2}-x^{2} \Delta^{2}} \mathcal{R}\left(\frac{x \Delta}{m_{X}}\right)\right. \\
& \left.+\mathcal{C}(D-F) \sum_{X} \beta_{X}^{B} \int_{0}^{1} d x x(1-x)\left(x-\frac{1}{2}\right) \frac{\Delta m_{X}}{m_{X}^{2}-x^{2} \Delta^{2}} \mathcal{R}\left(-\frac{x \Delta}{m_{X}}\right)\right],
\end{aligned}
$$

where the function $\mathcal{R}(x)$ is given by

$$
\mathcal{R}(x)=\sqrt{x^{2}-1} \log \frac{x-\sqrt{x^{2}-1+i \epsilon}}{x+\sqrt{x^{2}-1+i \epsilon}}
$$

and we have only kept loop contributions that are non-analytic in the meson mass $m_{X}$. The tree-level coefficients $\alpha$ are listed in Table $\square$ and the coefficients for the loop diagrams in Fig. (2), $\beta_{X}^{T}$ and $\beta_{X}^{B}$, are given in Tables [I and [II respectively. In these tables we have listed values corresponding to the loop meson with mass $m_{X}$. As required, in the QCD limit the PQ $\chi \mathrm{PT}$ coefficients reduce to those of $\chi \mathrm{PT}$. It is comforting that the one-loop results for the $G_{3}\left(q^{2}\right)$ form factor are finite. This is consistent with the fact that one cannot write down a dimension-7 operator that contributes at the same order in the chiral expansion as our one-loop result for $G_{3}\left(q^{2}\right)$. The full one-loop $q^{2}$ dependence of these form factors can easily be recovered by replacing

$$
m_{X} \rightarrow \sqrt{m_{X}^{2}-x(1-x) q^{2}} .
$$

Notice that the tree-level transitions $\Sigma^{*,-} \rightarrow \Sigma^{-} \gamma$ and $\Xi^{*,-} \rightarrow \Xi^{-} \gamma$ are zero because they are forbidden by $d \leftrightarrow s U$-spin symmetry [49]. There is also symmetry between the $\Sigma^{*,+} \rightarrow \Sigma^{+} \gamma$ and $\Xi^{*, 0} \rightarrow \Xi^{0} \gamma$ transitions as well as the $\Sigma^{*,-} \rightarrow \Sigma^{-} \gamma$ and $\Xi^{*,-} \rightarrow \Xi^{-} \gamma$ transitions that holds to NLO in $\chi \mathrm{PT}$ and PQ $\chi \mathrm{PT}$.

\section{2. $Q \chi P T$}

The calculation of the transition moments can be repeated in $\mathrm{Q} \chi \mathrm{PT}$. At tree level, the operators in Eq. (20) contribute, but their low-energy coefficients cannot be matched onto 
TABLE II: The $S U(3)$ coefficients $\beta_{X}^{T}$ in $\chi \mathrm{PT}$ and PQ $\chi \mathrm{PT}$.

\begin{tabular}{|c|c|c|c|c|c|c|c|c|c|}
\hline & \multicolumn{2}{|c|}{$\chi \mathrm{PT}$} & \multicolumn{7}{|c|}{$\mathrm{PQ} \chi \mathrm{PT}$} \\
\hline & $\pi$ & $K$ & $\pi$ & $K$ & $\eta_{s}$ & $j u$ & $r u$ & $j s$ & $r s$ \\
\hline$\Delta \rightarrow N \gamma$ & $\frac{5}{3 \sqrt{3}}$ & $\frac{1}{3 \sqrt{3}}$ & $\frac{1}{\sqrt{3}}$ & 0 & 0 & $\frac{2}{3 \sqrt{3}}$ & $\frac{1}{3 \sqrt{3}}$ & 0 & 0 \\
\hline$\Sigma^{*,+} \rightarrow \Sigma^{+} \gamma$ & $-\frac{1}{3 \sqrt{3}}$ & $\begin{array}{r}3 \sqrt{3} \\
-\frac{5}{3 \sqrt{3}}\end{array}$ & $\frac{1-3 q_{j l}}{9 \sqrt{3}}$ & $-\frac{11-3 q_{j l}+3 q_{r}}{9 \sqrt{3}}$ & $\frac{1+3 q_{r}}{9 \sqrt{3}}$ & $-\frac{4-3 q_{j l}}{9 \sqrt{3}}$ & $-\frac{2-3 q_{r}}{9 \sqrt{3}}$ & $-\frac{2+3 q_{j l}}{9 \sqrt{3}}$ & $-\frac{1+3 q_{r}}{9 \sqrt{3}}$ \\
\hline$\Sigma^{*, 0} \rightarrow \Sigma^{0} \gamma$ & 0 & $\frac{1}{\sqrt{3}}$ & $-\frac{1-3 q_{j l}}{9 \sqrt{3}}$ & $\frac{13-6 q_{j l}+6 q_{r}}{18 \sqrt{3}}$ & $-\frac{1+3 q_{r}}{9 \sqrt{3}}$ & $\frac{1-3 q_{j l}}{9 \sqrt{3}}$ & $\frac{1-6 q_{r}}{18 \sqrt{3}}$ & $\frac{2+3 q_{j l}}{9 \sqrt{3}}$ & $\frac{1+3 q_{r}}{9 \sqrt{3}}$ \\
\hline$\Sigma^{*, 0} \rightarrow \Lambda \gamma$ & $-\frac{2}{3}$ & $-\frac{1}{3}$ & $-\frac{1}{3}$ & $-\frac{1}{6}$ & 0 & $-\frac{1}{3}$ & $-\frac{1}{6}$ & 0 & 0 \\
\hline$\Sigma^{*,-} \rightarrow \Sigma^{-} \gamma$ & $-\frac{1}{1}$ & $\frac{1}{0}$ & $-\frac{1-3 q_{j l}}{\sqrt{5}}$ & $\frac{2-3 q_{j l}+3 q_{r}}{2}$ & $-\underline{1+3 q_{r}}$ & $-2+3 q_{j l}$ & $\begin{array}{r}1+3 q_{r} \\
-\underline{r}\end{array}$ & $\underline{2+3 q_{j l}}$ & $\underline{1+3 q_{r}}$ \\
\hline$\Xi^{*, 0} \rightarrow \Xi^{0} \gamma$ & $\begin{array}{r}3 \sqrt{3} \\
-\quad 1 \\
\end{array}$ & $\begin{array}{r}3 \sqrt{3} \\
-\quad 5 \\
\end{array}$ & $\begin{array}{r}9 \sqrt{3} \\
1-3 q_{j l} \\
\end{array}$ & $\begin{array}{c}9 \sqrt{3} \\
-11-3 q_{j l}+3 q_{r}\end{array}$ & $\begin{array}{r}9 \sqrt{3} \\
1+3 q_{r} \\
\end{array}$ & $\begin{array}{r}9 \sqrt{3} \\
-4-3 q_{j l} \\
\end{array}$ & $\begin{array}{r}9 \sqrt{3} \\
-2-3 q_{r} \\
\end{array}$ & $\begin{array}{l}9 \sqrt{3} \\
2+3 q_{j l}\end{array}$ & $\begin{array}{l}9 \sqrt{3} \\
1+3 q_{r}\end{array}$ \\
\hline$\exists \rightarrow=\gamma$ & $-\overline{3 \sqrt{3}}$ & $-\overline{3 \sqrt{3}}$ & $\begin{array}{l}9 \sqrt{3} \\
1-3 q_{i l}\end{array}$ & $\frac{9 \sqrt{3}}{2-3 q_{j l}+3 q_{r}}$ & $\begin{array}{l}9 \sqrt{3} \\
1+3 q_{r}\end{array}$ & $\begin{array}{c}\frac{9 \sqrt{3}}{2+3 q_{j l}}\end{array}$ & $\begin{array}{c}-\frac{\sqrt{3}}{1+3 q_{r}}\end{array}$ & $\begin{array}{r}-\frac{9 \sqrt{3}}{2+3 q_{i l}}\end{array}$ & $-\frac{\sqrt{3}}{9 \sqrt{3}}$ \\
\hline$\Xi^{*,-} \rightarrow \Xi^{-} \gamma$ & $-\frac{1}{3 \sqrt{3}}$ & $\frac{1}{3 \sqrt{3}}$ & $\frac{1-5 q_{j} l}{9 \sqrt{3}}$ & $\frac{2-5 q_{j} l+5 q r}{9 \sqrt{3}}$ & $-\frac{1+3 q_{r}}{9 \sqrt{3}}$ & $-\frac{2+9 q_{j} l}{9 \sqrt{3}}$ & $-\frac{1+3 q_{r}}{9 \sqrt{3}}$ & $\frac{2+0 q_{j l} l}{9 \sqrt{3}}$ & $\frac{1+3 q_{r}}{9 \sqrt{3}}$ \\
\hline
\end{tabular}


TABLE III: The $S U(3)$ coefficients $\beta_{X}^{B}$ in $\chi \mathrm{PT}$ and PQ $\chi \mathrm{PT}$.

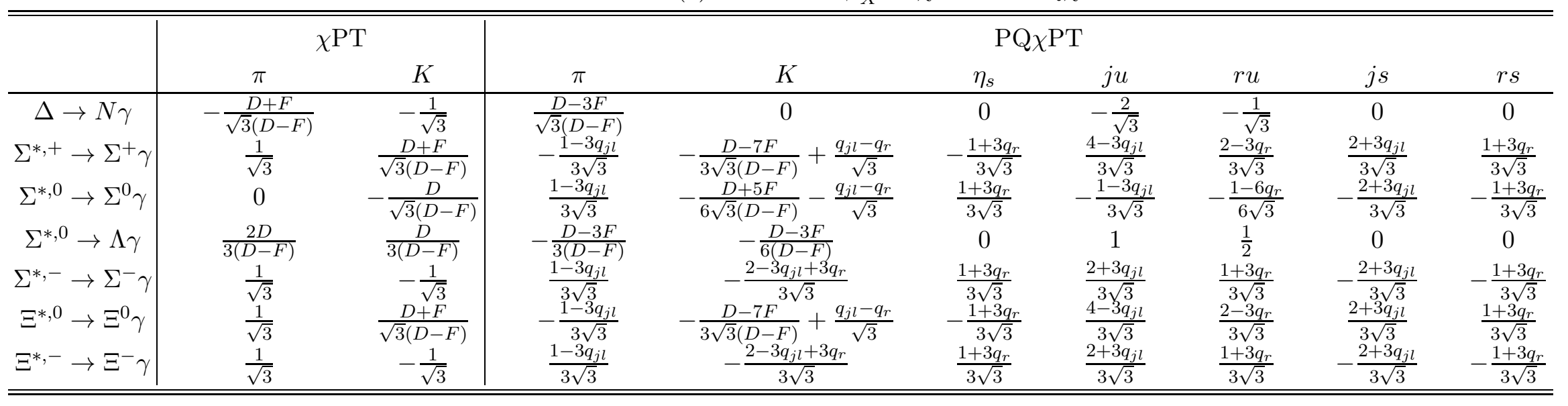


TABLE IV: The $S U(3)$ coefficients $\beta_{X}^{B, Q}$ and $\beta_{X}^{T, Q}$ in $\mathrm{Q} \chi \mathrm{PT}$.

\begin{tabular}{c|cc|cc} 
& \multicolumn{2}{|c|}{$\beta_{X}^{T, Q}$} & \multicolumn{3}{|c}{$\beta_{X}^{B, Q}$} \\
& $\pi$ & $K$ & $\pi$ & $K$ \\
\hline$\Delta \rightarrow N \gamma$ & $\frac{1}{\sqrt{3}}$ & 0 & $\frac{1}{\sqrt{3}}\left(D^{Q}-3 F^{Q}\right)$ & 0 \\
$\Sigma^{*,+} \rightarrow \Sigma^{+} \gamma$ & 0 & $-\frac{1}{\sqrt{3}}$ & 0 & $-\frac{1}{\sqrt{3}}\left(D^{Q}-3 F^{Q}\right)$ \\
$\Sigma^{*, 0} \rightarrow \Sigma^{0} \gamma$ & 0 & $\frac{1}{2 \sqrt{3}}$ & 0 & $\frac{1}{2 \sqrt{3}}\left(D^{Q}-3 F^{Q}\right)$ \\
$\Sigma^{*, 0} \rightarrow \Lambda \gamma$ & $-\frac{1}{3}$ & $-\frac{1}{6}$ & $-\frac{1}{3}\left(D^{Q}-3 F^{Q}\right)$ & $-\frac{1}{6}\left(D^{Q}-3 F^{Q}\right)$ \\
$\Sigma^{*,-} \rightarrow \Sigma^{-} \gamma$ & 0 & 0 & 0 & 0 \\
$\Xi^{*, 0} \rightarrow \Xi^{0} \gamma$ & 0 & $-\frac{1}{\sqrt{3}}$ & 0 & $-\frac{1}{\sqrt{3}}\left(D^{Q}-3 F^{Q}\right)$ \\
$\Xi^{*,-} \rightarrow \Xi^{-} \gamma$ & 0 & 0 & 0 & 0
\end{tabular}
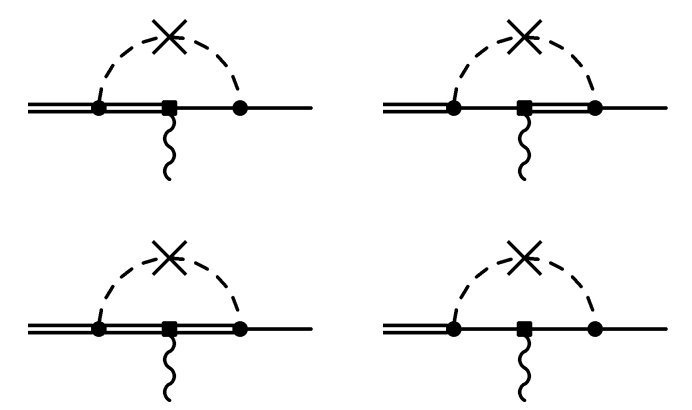

FIG. 3: Loop diagrams contributing to the transition form factors in $\mathrm{Q} \chi \mathrm{PT}$. The four diagrams correspond to terms involving the parameters $A_{X X^{\prime}}, B_{X X^{\prime}}, C_{X X^{\prime}}$, and $D_{X X^{\prime}}$ in Eqs. (29) and (30).

QCD. Therefore we annotate them with a "Q". At the next order in the chiral expansion, there are again contributions from the loop diagrams in Fig. (2). The results are the same as in the partially quenched theory, Eqs. (21) -(23), with the coefficients $\beta_{X}^{T}$ and $\beta_{X}^{B}$ replaced by $\beta_{X}^{T, Q}$ and $\beta_{X}^{B, Q} /\left(D^{Q}-F^{Q}\right)$, which are listed in Table IV

In addition, there are contributions of the form $\mu_{0}^{2} \log m_{q}$ at the same order in the chiral expansion that are artifacts of quenching. These come from hairpin wavefunction renormalization diagrams and from the four loop diagrams in Fig. (3). In these diagrams the photon can couple to the baryon line via

$$
\begin{aligned}
\mathcal{L}= & \frac{i e}{2 M_{B}}\left[\mu_{\alpha}^{Q}\left(\overline{\mathcal{B}}\left[S_{\mu}, S_{\nu}\right] \mathcal{B} \mathcal{Q}\right)+\mu_{\beta}^{Q}\left(\overline{\mathcal{B}}\left[S_{\mu}, S_{\nu}\right] \mathcal{Q B}\right)\right] F^{\mu \nu} \\
& +\mu_{c}^{Q} \frac{3 i e}{M_{B}}\left(\overline{\mathcal{T}}_{\mu} \mathcal{Q} \mathcal{T}_{\nu}\right) F^{\mu \nu}-\mathbb{Q}_{c}^{Q} \frac{3 e}{\Lambda_{\chi}^{2}}\left(\overline{\mathcal{T}}^{\{\mu} \mathcal{Q} \mathcal{T}^{\nu\}}\right) v^{\alpha} \partial_{\mu} F_{\nu \alpha}
\end{aligned}
$$

and via the terms in Eq. (20) including their hermitian conjugates (with quenched coeffi- 
cients). ${ }^{3}$ It is easier to work with the combinations $\mu_{D}^{Q}$ and $\mu_{F}^{Q}$ defined by

$$
\mu_{\alpha}^{Q}=\frac{2}{3} \mu_{D}^{Q}+2 \mu_{F}^{Q} \quad \text { and } \quad \mu_{\beta}^{Q}=-\frac{5}{3} \mu_{D}^{Q}+\mu_{F}^{Q}
$$

Although the argument presented in [50] does not apply to the case of different initial and final states, the axial hairpin interactions still do not contribute simply because their presence requires closed quark loops. The hairpin wavefunction renormalization diagrams have been calculated in $\mathrm{Q} \chi \mathrm{PT}$ for the baryon octet [15] $\left(Z_{B}^{Q}\right)$ and decuplet [18] $\left(Z_{T}^{Q}\right)$ and we do not reproduce them here. We find the hairpin contributions to the transition form factors to be

$$
\begin{aligned}
G_{1}^{H P}\left(q^{2}\right)= & \frac{\mu_{T}^{Q}}{2} \alpha \frac{Z_{B}^{Q}-1}{2} \frac{Z_{T}^{Q}-1}{2} \\
+ & \frac{\mu_{0}^{2}}{16 \pi^{2} f^{2}} \sum_{X, X^{\prime}}\left[\frac{5}{108} \mathcal{H}^{Q} \mu_{T}^{Q} A_{X X^{\prime}} I_{X X^{\prime}}-\frac{1}{18}\left(\mathcal{C}^{Q}\right)^{2} \mu_{T}^{Q} B_{X X^{\prime}} I_{X X^{\prime}}^{-\Delta, \Delta}\right. \\
& \left.\quad-\frac{20}{27} \mathcal{H}^{Q} \mathcal{C}^{Q} Q_{T} \mu_{c}^{Q} C_{X X^{\prime}} I_{X X^{\prime}}^{\Delta}-\frac{2}{3} \mathcal{C}^{Q}\left(Q_{T} \mu_{F}^{Q}+\alpha_{D} \mu_{D}^{Q}\right) D_{X X^{\prime}} I_{X X^{\prime}}^{\Delta}\right] \\
G_{2}^{H P}\left(q^{2}\right)= & -4 \mathbb{Q}_{T}^{Q} \alpha \frac{M_{B}^{2}}{\Lambda_{\chi}^{2}} \frac{Z_{B}^{Q}-1}{2} \frac{Z_{T}^{Q}-1}{2} \\
& +\frac{\mu_{0}^{2}}{16 \pi^{2} f^{2}} \frac{M_{B}^{2}}{\Lambda_{\chi}^{2}} \sum_{X X^{\prime}}\left[\frac{2}{9} \mathcal{H}^{Q} \mathbb{Q}_{T}^{Q} A_{X X^{\prime}} I_{X X^{\prime}}+\frac{4}{3}\left(C^{Q}\right)^{2} \mathbb{Q}_{T}^{Q} B_{X X^{\prime}} I_{X X^{\prime}}^{-\Delta \Delta}\right. \\
& \left.-\frac{16}{9} \mathcal{H}^{Q} \mathcal{C}^{Q} Q_{T} \mathbb{Q}_{C}^{Q} C_{X X^{\prime}} I_{X X^{\prime}}^{\Delta}\right]
\end{aligned}
$$

and $G_{3}^{H P}\left(q^{2}\right)=0$. Thus in $\mathrm{Q} \chi \mathrm{PT}: G_{j}^{Q}\left(q^{2}\right)=G_{j}^{P Q}\left(q^{2}\right)+G_{j}^{H P}\left(q^{2}\right)$, where the $\beta_{X}^{T}$ and $\beta_{X}^{B}$ coefficients of $G_{j}^{P Q}\left(q^{2}\right)$, Eqs. (21)-(23), are understood to be replaced by their quenched values $\beta_{X}^{T, Q}$ and $\beta_{X}^{B, Q} /\left(D^{Q}-F^{Q}\right)$. Above we have used the shorthand notation $I_{\eta_{q} \eta_{q^{\prime}}}=$ $I\left(m_{\eta_{q}}, m_{\eta_{q^{\prime}}}, 0,0, \mu\right), I_{\eta_{q} \eta_{q^{\prime}}}^{\Delta}=I\left(m_{\eta_{q}}, m_{\eta_{q^{\prime}}}, \Delta, 0, \mu\right)$, and $I_{\eta_{q} \eta_{q^{\prime}}}^{\Delta_{1}, \Delta_{2}}=I\left(m_{\eta_{q}}, m_{\eta_{q^{\prime}}}, \Delta_{1}, \Delta_{2}, \mu\right)$ for the function $I\left(m_{1}, m_{2}, \Delta_{1}, \Delta_{2}, \mu\right)$ that is given by

$$
I\left(m_{1}, m_{2}, \Delta_{1}, \Delta_{2}, \mu\right)=\frac{Y\left(m_{1}, \Delta_{1}, \mu\right)+Y\left(m_{2}, \Delta_{2}, \mu\right)-Y\left(m_{1}, \Delta_{2}, \mu\right)-Y\left(m_{2}, \Delta_{1}, \mu\right)}{\left(m_{1}^{2}-m_{2}^{2}\right)\left(\Delta_{1}-\Delta_{2}\right)}
$$

with

$$
Y(m, \Delta, \mu)=\Delta\left(m^{2}-\frac{2}{3} \Delta^{2}\right) \log \frac{m^{2}}{\mu^{2}}+\frac{2}{3} m\left(\Delta^{2}-m^{2}\right) \mathcal{R}\left(\frac{\Delta}{m}\right)
$$

${ }^{3}$ Note that possible contributions from diagrams involving

$$
\mathcal{L}=\frac{e}{\Lambda_{\chi}^{2}}\left[c_{\alpha}^{Q}(\overline{\mathcal{B}} \mathcal{B} \mathcal{Q})+c_{\beta}^{Q}(\overline{\mathcal{B}} \mathcal{Q B})\right] v_{\mu} \partial_{\nu} F^{\mu \nu}+c_{c}^{Q} \frac{3 e}{\Lambda_{\chi}^{2}}\left(\overline{\mathcal{T}}^{\sigma} \mathcal{Q} \mathcal{T}_{\sigma}\right) v_{\mu} \partial_{\nu} F^{\mu \nu}
$$

are identically zero. 
TABLE V: The $S U(3)$ coefficients $A_{X X^{\prime}}$ and $B_{X X^{\prime}}$ in $\mathrm{Q} \chi \mathrm{PT}$.

\begin{tabular}{c|ccc|ccc}
\hline \hline & \multicolumn{3}{c|}{$A_{X X^{\prime}}$} & \multicolumn{3}{c}{$B_{X X^{\prime}}$} \\
& $\eta_{u} \eta_{u}$ & $\eta_{u} \eta_{s}$ & $\eta_{s} \eta_{s}$ & $\eta_{u} \eta_{u}$ & $\eta_{u} \eta_{s}$ & $\eta_{s} \eta_{s}$ \\
\hline$\Delta \rightarrow N \gamma$ & $2 \sqrt{3}\left(D^{Q}-3 F^{Q}\right)$ & 0 & 0 & 0 & 0 & 0 \\
$\Sigma^{*,+} \rightarrow \Sigma^{+} \gamma$ & $\frac{8}{\sqrt{3}} F^{Q}$ & $-\frac{4}{\sqrt{3}}\left(D^{Q}-2 F^{Q}\right)$ & $-\frac{2}{\sqrt{3}}\left(D^{Q}-F^{Q}\right)$ & $\frac{1}{3 \sqrt{3}}$ & $-\frac{2}{3 \sqrt{3}}$ & $\frac{1}{3 \sqrt{3}}$ \\
$\Sigma^{*, 0} \rightarrow \Sigma^{0} \gamma$ & $-\frac{4}{\sqrt{3}} F^{Q}$ & $\frac{2}{\sqrt{3}}\left(D^{Q}-2 F^{Q}\right)$ & $\frac{1}{\sqrt{3}}\left(D^{Q}-F^{Q}\right)$ & $-\frac{1}{6 \sqrt{3}}$ & $\frac{1}{3 \sqrt{3}}$ & $-\frac{1}{6 \sqrt{3}}$ \\
$\Sigma^{*, 0} \rightarrow \Lambda \gamma$ & $-\frac{4}{3}\left(2 D^{Q}-3 F^{Q}\right)$ & $-\frac{2}{3}\left(D^{Q}-6 F^{Q}\right)$ & $\frac{1}{3}\left(D^{Q}+3 F^{Q}\right)$ & 0 & 0 & 0 \\
$\Sigma^{*,-} \rightarrow \Sigma^{-} \gamma$ & 0 & 0 & 0 & 0 & 0 & 0 \\
$\Xi^{*, 0} \rightarrow \Xi^{0} \gamma$ & $-\frac{2}{\sqrt{3}}\left(D^{Q}-F^{Q}\right)$ & $-\frac{4}{\sqrt{3}}\left(D^{Q}-2 F^{Q}\right)$ & $\frac{8}{\sqrt{3}} F^{Q}$ & $\frac{1}{3 \sqrt{3}}$ & $-\frac{2}{3 \sqrt{3}}$ & $\frac{1}{3 \sqrt{3}}$ \\
$\Xi^{*,-} \rightarrow \Xi^{-} \gamma$ & 0 & 0 & 0 & 0 & 0 & 0 \\
\hline \hline
\end{tabular}

TABLE VI: The $S U(3)$ coefficients $C_{X X^{\prime}}$ and $D_{X X^{\prime}}$ in $\mathrm{Q} \chi \mathrm{PT}$.

\begin{tabular}{c|ccc|ccc}
\hline \hline & \multicolumn{3}{|c|}{$C_{X X^{\prime}}$} & \multicolumn{3}{c}{$D_{X X^{\prime}}$} \\
& $\eta_{u} \eta_{u}$ & $\eta_{u} \eta_{s}$ & $\eta_{s} \eta_{s}$ & $\eta_{u} \eta_{u}$ & $\eta_{u} \eta_{s}$ & $\eta_{s} \eta_{s}$ \\
\hline$\Delta \rightarrow N \gamma$ & 0 & 0 & 0 & 0 & 0 & 0 \\
$\Sigma^{*,+} \rightarrow \Sigma^{+} \gamma$ & $-\frac{2}{3 \sqrt{3}}$ & $\frac{1}{3 \sqrt{3}}$ & $\frac{1}{3 \sqrt{3}}$ & $-\frac{2}{\sqrt{3}} F^{Q}$ & $\frac{1}{\sqrt{3}}\left(D^{Q}+F^{Q}\right)$ & $-\frac{1}{\sqrt{3}}\left(D^{Q}-F^{Q}\right)$ \\
$\Sigma^{*, 0} \rightarrow \Sigma^{0} \gamma$ & 0 & 0 & 0 & $\frac{2}{\sqrt{3}} F^{Q}$ & $-\frac{1}{\sqrt{3}}\left(D^{Q}+F^{Q}\right)$ & $\frac{1}{\sqrt{3}}\left(D^{Q}-F^{Q}\right)$ \\
$\Sigma^{*, 0} \rightarrow \Lambda \gamma$ & 0 & 0 & 0 & $-\frac{4}{3} D^{Q}+2 F^{Q}$ & $\frac{5}{3} D^{Q}-F^{Q}$ & $-\frac{1}{3} D^{Q}-F^{Q}$ \\
$\Sigma^{*,-} \rightarrow \Sigma^{-} \gamma$ & $\frac{2}{3 \sqrt{3}}$ & $-\frac{1}{3 \sqrt{3}}$ & $-\frac{1}{3 \sqrt{3}}$ & $\frac{2}{\sqrt{3}} F^{Q}$ & $-\frac{1}{\sqrt{3}}\left(D^{Q}+F^{Q}\right)$ & $\frac{1}{\sqrt{3}}\left(D^{Q}-F^{Q}\right)$ \\
$\Xi^{*, 0} \rightarrow \Xi^{0} \gamma$ & 0 & 0 & 0 & $\frac{1}{\sqrt{3}}\left(D^{Q}-F^{Q}\right)$ & $-\frac{1}{\sqrt{3}}\left(D^{Q}+F^{Q}\right)$ & $\frac{2}{\sqrt{3}} F^{Q}$ \\
$\Xi^{*,-} \rightarrow \Xi^{-} \gamma$ & $\frac{1}{3 \sqrt{3}}$ & $\frac{1}{3 \sqrt{3}}$ & $-\frac{2}{3 \sqrt{3}}$ & $-\frac{1}{\sqrt{3}}\left(D^{Q}-F^{Q}\right)$ & $\frac{1}{\sqrt{3}}\left(D^{Q}+F^{Q}\right)$ & $-\frac{2}{\sqrt{3}} F^{Q}$ \\
\hline \hline
\end{tabular}

The coefficients $A_{X X^{\prime}}, B_{X X^{\prime}}, C_{X X^{\prime}}$, and $D_{X X^{\prime}}$ are listed in Tables $\nabla$ and $\nabla \mathbf{D}$. Note that the symmetry between the $\Sigma^{*,+} \rightarrow \Sigma^{+} \gamma$ and $\Xi^{*, 0} \rightarrow \Xi^{0} \gamma$ transitions as well as the $\Sigma^{*,-} \rightarrow \Sigma^{-} \gamma$ and $\Xi^{*,-} \rightarrow \Xi^{-} \gamma$ transitions that holds in $\chi \mathrm{PT}$ and $\mathrm{PQ} \chi \mathrm{PT}$ is now broken by singlet loop contributions.

\section{CONCLUSIONS}

We have calculated the baryon octet to decuplet transition form factors in $\mathrm{Q} \chi \mathrm{PT}$ and PQ $\chi$ PT using the the isospin limit of $S U(3)$ flavor and have also derived the result for the nucleon doublet in two flavor PQ $\chi \mathrm{PT}$ away from the isospin limit. Extrapolating lattice calculations that employ the quenched or partially quenched approximation can only be done by using their respective low-energy theories, $\mathrm{Q} \chi \mathrm{PT}$ and $\mathrm{PQ} \chi \mathrm{PT}$. Whereas PQQCD can be smoothly connected to QCD, QQCD exhibits pathological behavior, in particular QQCD observables are usually more divergent in the chiral limit than in QCD. This stems from the fact that new operators not present in QCD must be included in the QQCD Lagrangian.

For the decuplet to octet transition form factors our NLO Q $\chi \mathrm{PT}$ results are not more divergent than their $\chi \mathrm{PT}$ counterparts: $G_{1}, G_{2} \sim \alpha+\beta \log m_{Q}$ and $G_{3} \sim \alpha$. This, however, does not mean that this result is free of quenching artifacts. The quenched transition 
moments pick up contributions from hairpin loops. A particular oddity is that the quark mass dependence of the $\Sigma^{*,-}$ and $\Xi^{*,-}$ quenched transition moments is solely due to the singlet parameter $\mu_{0}^{2}$; even worse, $G_{3}^{Q}\left(q^{2}\right)=0$ at this order. These transitions thus present extremes of the quenched approximation in agreement with the quenched lattice data of [5] where the $\Sigma^{*,-}$ and $\Xi^{*,-}$ E2 moments were found to be significantly different from the other transitions. In contrast to $\mathrm{Q} \chi \mathrm{PT}$ results, our $\mathrm{PQ} \chi \mathrm{PT}$ results will enable not only the extrapolation of PQQCD lattice simulations of the transition moments but also the extraction of predictions for the real world: QCD.

\section{Acknowledgments}

We would like to thank Martin Savage for very helpful discussions and for useful comments on the manuscript. This work is supported in part by the U.S. Department of Energy under Grant No. DE-FG03-97ER4014.

\section{APPENDIX: $\quad \Delta \rightarrow N \gamma$ TRANSITIONS IN $S U(2)$ FLAVOR WITH NON- DEGENERATE QUARKS}

In this Appendix, we repeat the calculation of the transition moments for the case of $S U(2)$ flavor with non-degenerate quarks, i.e., the quark mass matrix reads $m_{Q}^{S U(2)}=$ $\operatorname{diag}\left(m_{u}, m_{d}, m_{j}, m_{l}, m_{u}, m_{d}\right)$. Since defining ghost and sea quark charges is constrained only by the restriction that QCD be recovered in the limit of appropriately degenerate quark masses, the most general form of the charge matrix is

$$
\mathcal{Q}^{S U(2)}=\operatorname{diag}\left(\frac{2}{3},-\frac{1}{3}, q_{j}, q_{l}, q_{j}, q_{l}\right)
$$

The symmetry breaking pattern is assumed to be $S U(4 \mid 2)_{L} \otimes S U(4 \mid 2)_{R} \otimes U(1)_{V} \longrightarrow$ $S U(4 \mid 2)_{V} \otimes U(1)_{V}$. The baryon field assignments are analogous to the case of $S U(3)$ flavor. The nucleons are embedded as

$$
\mathcal{B}_{i j k}=\frac{1}{\sqrt{6}}\left(\epsilon_{i j} N_{k}+\epsilon_{i k} N_{j}\right)
$$

where the indices $i, j$ and $k$ are restricted to 1 or 2 and the $S U(2)$ nucleon doublet is defined as

$$
N=\left(\begin{array}{l}
p \\
n
\end{array}\right)
$$

The decuplet field $\mathcal{T}_{i j k}$, which is totally symmetric, is normalized to contain the $\Delta$-resonances $T_{i j k}=\mathcal{T}_{i j k}$ with $i, j, k$ restricted to 1 or 2 and $\mathcal{T}_{111}=\Delta^{++}$. The construction of the octet and decuplet baryons containing one sea or one ghost quark is analogous to the $S U(3)$ flavor case [29] and will not be repeat here.

The free Lagrangian for $\mathcal{B}$ and $\mathcal{T}$ is the one in Eq. (13) (with the parameters having different numerical values than the $S U(3)$ case). The connection to QCD is detailed in [29]. Similarly, the Lagrangian describing the interaction of the $\mathcal{B}$ and $\mathcal{T}$ with the pseudo-Goldstone 
TABLE VII: The $S U(2)$ coefficients $\beta_{X}^{B}$ and $\beta_{X}^{T}$ in PQ $\chi$ PT for $\Delta \rightarrow N \gamma$.

\begin{tabular}{c|cc}
\hline \hline & $\beta_{X}^{B}$ & $\beta_{X}^{T}$ \\
\hline$u u$ & $\frac{1}{3 \sqrt{3}}\left(2-3 q_{j}\right)$ & $-\frac{1}{9 \sqrt{3}}\left(2-3 q_{j}\right)$ \\
$u d$ & $\frac{1}{\sqrt{3}}\left[1+q_{j}-q_{l}+2 \frac{g_{A}}{g_{1}}\right]$ & $\frac{1}{3 \sqrt{3}}\left(4-q_{j}+q_{l}\right)$ \\
$d d$ & $\frac{1}{3 \sqrt{3}}\left(1+3 q_{l}\right)$ & $-\frac{1}{9 \sqrt{3}}\left(1+3 q_{l}\right)$ \\
$j u$ & $-\frac{1}{3 \sqrt{3}}\left(2-3 q_{j}\right)$ & $\frac{1}{9 \sqrt{3}}\left(2-3 q_{j}\right)$ \\
$l u$ & $-\frac{1}{3 \sqrt{3}}\left(2-3 q_{l}\right)$ & $\frac{1}{9 \sqrt{3}}\left(2-3 q_{l}\right)$ \\
$j d$ & $-\frac{1}{3 \sqrt{3}}\left(1+3 q_{j}\right)$ & $\frac{1}{9 \sqrt{3}}\left(1+3 q_{j}\right)$ \\
$l d$ & $-\frac{1}{3 \sqrt{3}}\left(1+3 q_{l}\right)$ & $\frac{1}{9 \sqrt{3}}\left(1+3 q_{l}\right)$ \\
\hline \hline
\end{tabular}

bosons is the one in Eq. (14) that can be matched to the familiar one in QCD (by restricting the $\mathcal{B}_{i j k}$ and $\mathcal{T}_{i j k}$ to the $q q q$ sector),

$$
\begin{aligned}
\mathcal{L}= & 2 g_{A} \bar{N} S^{\mu} A_{\mu} N+g_{1} \bar{N} S^{\mu} N \operatorname{tr}\left(A_{\mu}\right)+g_{\Delta N}\left(\bar{T}_{\nu}^{k j i} A_{i l}^{\nu} N_{j} \epsilon_{k l}+\text { h.c }\right) \\
& +2 g_{\Delta \Delta} \bar{T}_{k j i}^{\nu} S_{\mu} A_{i l}^{\mu} T_{\nu, l j k}+2 g_{X} \bar{T}_{k j i}^{\nu} S_{\mu} T_{\nu, i j k} \operatorname{tr}\left(A^{\mu}\right),
\end{aligned}
$$

where one finds at tree-level $g_{1}=-2(D-F), g_{A}=D+F, \mathcal{C}=-g_{\Delta N}$, and $\mathcal{H}=g_{\Delta \Delta}$, with $g_{X}=0$. The leading tree-level operators which contribute to $\Delta \rightarrow N \gamma$ have the same form as in Eq. (20), of course the low-energy constants have different values.

Evaluating the transition moments at NLO in the chiral expansion yields expressions identical in form to those in Eqs. (21) - (23) with the $\mathrm{SU}(2)$ identifications made for $\mathcal{C}, \mathcal{H}$, $D$, and $F$. For the $S U(2)$ coefficients in $\chi \mathrm{PT}$ one finds $\beta_{X}^{B}=g_{A} / \sqrt{3}$ and $\beta_{X}^{T}=5 /(3 \sqrt{3})$ for the $\pi^{ \pm}$. The corresponding values for the case of PQ $\chi \mathrm{PT}$ appear in Table VII.

[1] C. Mertz et al., Phys. Rev. Lett. 86, 2963 (2001), nucl-ex/9902012.

[2] K. Joo et al. (CLAS), Phys. Rev. Lett. 88, 122001 (2002), hep-ex/0110007.

[3] C. Alexandrou et al. (2002), hep-lat/0209074.

[4] C. Alexandrou et al. (2003), hep-lat/0307018.

[5] D. B. Leinweber, T. Draper, and R. M. Woloshyn, Phys. Rev. D48, 2230 (1993), heplat/9212016.

[6] A. Morel, J. Phys. (France) 48, 1111 (1987).

[7] S. R. Sharpe, Phys. Rev. D46, 3146 (1992), hep-lat/9205020.

[8] C. W. Bernard and M. Golterman, Nucl. Phys. Proc. Suppl. 26, 360 (1992).

[9] C. W. Bernard and M. F. L. Golterman, Phys. Rev. D46, 853 (1992), hep-lat/9204007.

[10] M. F. L. Golterman, Acta Phys. Polon. B25, 1731 (1994), hep-lat/9411005.

[11] S. R. Sharpe and Y. Zhang, Phys. Rev. D53, 5125 (1996), hep-lat/9510037.

[12] J. N. Labrenz and S. R. Sharpe, Phys. Rev. D54, 4595 (1996), hep-lat/9605034.

[13] M. J. Booth (1994), hep-ph/9412228.

[14] M. Kim and S. Kim, Phys. Rev. D58, 074509 (1998), hep-lat/9608091.

[15] M. J. Savage, Nucl. Phys. A700, 359 (2002), nucl-th/0107038.

[16] D. Arndt, Phys. Rev. D67, 074501 (2003), hep-lat/0210019.

[17] D. Arndt and B. C. Tiburzi (2003), hep-lat/0307003. 
[18] D. Arndt and B. C. Tiburzi (2003), hep-lat/0308001.

[19] S. R. Sharpe and N. Shoresh, Int. J. Mod. Phys. A16S1C, 1219 (2001), hep-lat/0011089.

[20] S. R. Sharpe and N. Shoresh, Phys. Rev. D64, 114510 (2001), hep-lat/0108003.

[21] C. W. Bernard and M. F. L. Golterman, Phys. Rev. D49, 486 (1994), hep-lat/9306005.

[22] S. R. Sharpe, Phys. Rev. D56, 7052 (1997), hep-lat/9707018.

[23] M. F. L. Golterman and K.-C. Leung, Phys. Rev. D57, 5703 (1998), hep-lat/9711033.

[24] S. R. Sharpe and N. Shoresh, Nucl. Phys. Proc. Suppl. 83, 968 (2000), hep-lat/9909090.

[25] S. R. Sharpe and N. Shoresh, Phys. Rev. D62, 094503 (2000), hep-lat/0006017.

[26] N. Shoresh (2001), Ph.D. thesis, UMI-30-36529.

[27] M. J. Savage, Phys. Rev. D65, 034014 (2002), hep-ph/0109190.

[28] J.-W. Chen and M. J. Savage, Phys. Rev. D65, 094001 (2002), hep-lat/0111050.

[29] S. R. Beane and M. J. Savage, Nucl. Phys. A709, 319 (2002), hep-lat/0203003.

[30] M. J. Savage (2002), hep-lat/0208022.

[31] D. B. Leinweber (2002), hep-lat/0211017.

[32] URL http://www.jlab.org/ dgr/lhpc/march00.pdf.

[33] URL http://www.jlab.org/ dgr/lhpc/sdac_proposal_final.pdf.

[34] S. R. Beane and M. J. Savage, Phys. Rev. D67, 054502 (2003), hep-lat/0210046.

[35] S. R. Beane and M. J. Savage, Phys. Lett. B535, 177 (2002), hep-lat/0202013.

[36] D. Arndt, S. R. Beane, and M. J. Savage (2003), nucl-th/0304004.

[37] E. Jenkins and A. V. Manohar, Phys. Lett. B255, 558 (1991).

[38] E. Jenkins and A. V. Manohar (1991), talk presented at the Workshop on Effective Field Theories of the Standard Model, Dobogoko, Hungary, Aug 1991.

[39] A. B. Balantekin, I. Bars, and F. Iachello, Phys. Rev. Lett. 47, 19 (1981).

[40] A. B. Balantekin and I. Bars, J. Math. Phys. 22, 1149 (1981).

[41] A. B. Balantekin and I. Bars, J. Math. Phys. 23, 1239 (1982).

[42] M. Golterman and E. Pallante, Nucl. Phys. Proc. Suppl. 106, 335 (2002), hep-lat/0110183.

[43] M. N. Butler, M. J. Savage, and R. P. Springer, Nucl. Phys. B399, 69 (1993), hep-ph/9211247.

[44] M. N. Butler, M. J. Savage, and R. P. Springer, Phys. Lett. B304, 353 (1993), hep-ph/9302214.

[45] M. Napsuciale and J. L. Lucio, Nucl. Phys. B494, 260 (1997), hep-ph/9609252.

[46] G. C. Gellas, T. R. Hemmert, C. N. Ktorides, and G. I. Poulis, Phys. Rev. D60, 054022 (1999), hep-ph/9810426.

[47] E. Jenkins, X.-d. Ji, and A. V. Manohar, Phys. Rev. Lett. 89, 242001 (2002), hep-ph/0207092.

[48] H. F. Jones and M. D. Scadron, Ann. Phys. 81, 1 (1973).

[49] H. J. Lipkin, Phys. Rev. D7, 846 (1973).

[50] C.-K. Chow, Phys. Rev. D57, 6762 (1998), hep-ph/9711375. 